\title{
Small-angle scattering standards and absolute intensity calibration
}

Andrew John Allen ${ }^{1}$, Fan Zhang ${ }^{1}$, R. Joseph Kline ${ }^{1}$, William F. Guthrie ${ }^{2}$, Jan Ilavsky ${ }^{3}$

${ }^{1}$ Material Measurement Lab., National Institute Of Standards \& Technology (NIST), Gaithersburg, United States, ${ }^{2}$ Information Technology Laboratory, National Institute of Standards and Technology, Gaithersburg, United States, ${ }^{3} X$-ray Science Division, Argonne National Laboratory, Argonne, United States E-mail: andrew.allen@nist.gov

Absolute intensity calibration of small-angle X-ray or neutron scattering (SAXS or SANS) data is a key requirement for the quantitative determination of volume fractions and surface areas of relevant nano and mesoscale inhomogeneities within advanced technological materials. Direct measurement of the scattering probability for a given sample requires calibration of the weak scattered-beam intensity relative to that of the incident beam. This calls for more than 8 decades in detector linear-intensity dynamic range, which is beyond the capabilities of typical 2D detectors employed in SAXS or SANS instruments. Various indirect calibration methods are used to overcome the issue, each with advantages and disadvantages. In practice, intensity calibration is performed "locally" for individual instruments or research institutes, with significant variations in calibrated results. For industrial laboratory-based SAXS instruments supporting biomedical, pharmaceutical or nanotechnology research, there may be no intensity calibration at all. We have addressed this issue by developing an absolute intensity calibration standard for SAXS, based on glassy carbon [1]. The new standard reference material (SRM) has been certified using the ultra-small-angle X-ray scattering (USAXS) facility at the Advanced Photon Source (APS), Argonne National Laboratory, Argonne, IL, USA [2]. Operating on a different principle to conventional 2D SAXS setups, the USAXS instrument achieves intensity calibration through direct measurements of the scattering probability with respect to the intensity of the incident X-ray beam. Users measure the SAXS intensity as a function of the scattering angle for both their samples and the glassy carbon SRM coupon; the intensity measured for the SRM is then compared to the certified calibration curve (see Figure) to extract a calibration factor which is applied to the sample data. The certified calibration data were obtained through USAXS measurements at the APS, supplemented by 2D SAXS measurements using a laboratorybased instrument at NIST, Gaithersburg, MD, USA, and were validated by SANS measurements at the NIST Center for Neutron Research (NCNR) [3]. The advantages of using such a calibration standard will be discussed in the context of smallangle scattering standards in general, and the increasing demand for data and intensity calibration to be portable between instruments and facilities.

[1] Zhang, F., Ilavsky, J., Long, G.G., Quintana, J., Allen, A.J., Jemian, P.R. (2010). Metall. Mater. Trans. A, 41, $1151-1158$.

[2] Ilavsky, J., Jemian, P.R., Allen, A.J., Zhang, F., Levine, L.E., Long, G.G. (2009). J.Appl.Cryst., 42, $469-479$.

[3] Allen, A.J., Zhang, F., Kline, R.J., Guthrie, W.F., Ilavsky, J. (2017). J. Appl. Cryst., 50, 462-474.

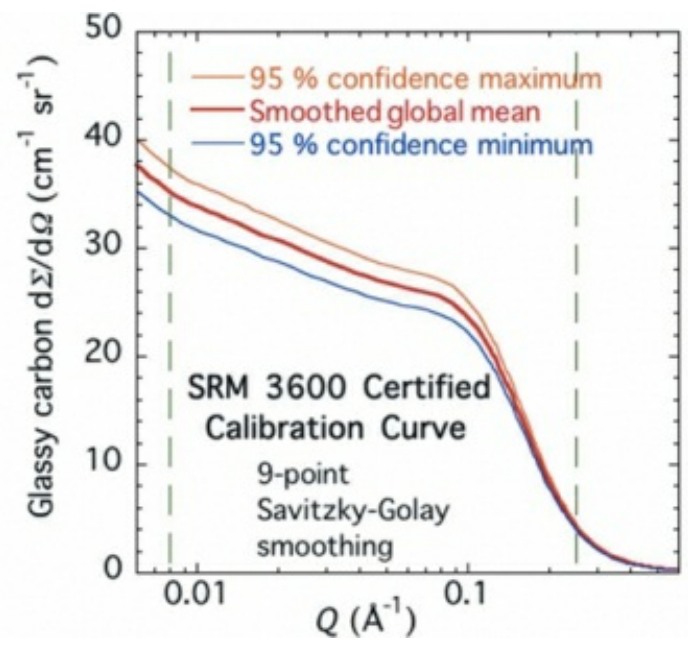

Keywords: small-angle scattering standards, absolute intensity calibration, data formats 